\title{
Off-Line Robust Constrained MPC for Linear Time-Varying Systems with Persistent Disturbances
}

\author{
P. Bumroongsri ${ }^{1}$ and S. Kheawhom ${ }^{2}$ \\ ${ }^{1}$ Department of Chemical Engineering, Faculty of Engineering, Mahidol University, Phuttamonthon 4 Road, \\ Salaya, Nakhon Pathom 73170, Thailand \\ ${ }^{2}$ Department of Chemical Engineering, Faculty of Engineering, Chulalongkorn University, Phayathai Road, \\ Pathumwan, Bangkok 10330, Thailand \\ Correspondence should be addressed to P. Bumroongsri; pornchai.bum@mahidol.ac.th
}

Received 13 November 2013; Revised 4 March 2014; Accepted 10 March 2014; Published 7 April 2014

Academic Editor: Driss Mehdi

Copyright (C) 2014 P. Bumroongsri and S. Kheawhom. This is an open access article distributed under the Creative Commons Attribution License, which permits unrestricted use, distribution, and reproduction in any medium, provided the original work is properly cited.

\begin{abstract}
An off-line robust constrained model predictive control (MPC) algorithm for linear time-varying (LTV) systems is developed. A novel feature is the fact that both model uncertainty and bounded additive disturbance are explicitly taken into account in the offline formulation of MPC. In order to reduce the on-line computational burdens, a sequence of explicit control laws corresponding to a sequence of positively invariant sets is computed off-line. At each sampling time, the smallest positively invariant set containing the measured state is determined and the corresponding control law is implemented in the process. The proposed MPC algorithm can guarantee robust stability while ensuring the satisfaction of input and output constraints. The effectiveness of the proposed MPC algorithm is illustrated by two examples.
\end{abstract}

\section{Introduction}

Model predictive control (MPC), also known as moving horizon control (MHC), is an advanced control algorithm that solves on-line a dynamic optimization problem based on an explicit model of the process. Although a sequence of control inputs is computed at each sampling instant, only the first computed input is implemented in the process. Since model is only an approximation of the real process, it is important for MPC to be robust to model uncertainty and disturbance [1-4].

The first method to tackle the problem of robustness is open-loop MPC [5]. At each sampling time, a sequence of control inputs is obtained by solving an optimal control problem in which model uncertainty is taken into account. The main drawback of this method lies in the fact that the use of open-loop predictions results in a significant spread of the predicted trajectories. For this reason, its implementation is only feasible for short prediction horizon. In order to overcome this problem, feedback MPC has been widely investigated. Kothare et al. [6] proposed a feedback MPC synthesis allowing explicit incorporation of model uncertainty in the problem formulation. The optimization problem at each sampling time is formulated as convex optimization problem subject to linear matrix inequality (LMI) constraints [7]. The extensions of this MPC algorithm were presented by Mao [8] and Wada et al. [9] where parameter-dependent Lyapunov functions were used instead of a single Lyapunov function. The conservativeness is reduced at the expense of a higher online computational complexity. These MPC algorithms are derived by assuming that there is no disturbance present so they can deal with only model uncertainty.

Another way to tackle the problem of robustness is a target set technique as proposed by Brooms et al. [10]. In the presence of model uncertainty, all predicted future states on the finite horizon are restricted to lie in a sequence of ellipsoidal sets. The terminal ellipsoidal set is contained in an ellipsoidal target set guaranteeing stability. This MPC algorithm is based on the idea of Lee and Kouvaritakis [11] where 
the polyhedral invariant sets are used in the controller design. For other methods that use polyhedral invariant sets in the MPC formulation, the reader is referred to Rossiter et al. [12], Pluymers et al. [13], and Bumroongsri and Kheawhom [14]. The main drawback of these MPC algorithms is that only model uncertainty is considered in the MPC formulation so they cannot deal with disturbance. The disturbance is explicitly considered in the synthesis of the state feedback control law in $[15,16]$. The proposed algorithm gives the best state feedback gain in terms of finding the maximal invariant ellipsoid. The concept of pole placement in LMI regions is introduced in order to improve the closed-loop performance. However, the value of the state feedback gain is not updated based on the information on new measurement at each sampling time so the conservativeness may be obtained.

In the context of robust tube-based MPC [17-19], the disturbance is directly taken into account in the problem formulation. The main idea is to compute the regions around a nominal predicted trajectory that contain all possible states of an uncertain system. The on-line computational complexity of these approaches increases with the prediction horizon.

In order to overcome the computational problems of MPC, current researchers have focused on how to reduce such a high computational burden while still ensuring the same level of control performance. An efficient off-line formulation of robust MPC was presented by Wan and Kothare [20]. The main idea is to solve the optimization problem offline to find a sequence of controller gains and the associated ellipsoidal invariant sets. The on-line computational effort can be reduced to a simple bisection search that determines the smallest ellipsoidal invariant set containing the measured state. An extension of this method has been developed by Ding et al. [21] where the nominal performance cost is used in the problem formulation instead of the worst-case performance cost. These off-line MPC algorithms can handle only model uncertainty and they cannot guarantee robust stability in the presence of disturbance.

Since there will always be some disturbances acting on the systems, they should be explicitly included in the controller design. In this research, an off-line robust constrained MPC algorithm for linear time-varying systems is presented. The main contribution is that both model uncertainty and bounded additive disturbance are explicitly taken into account in the off-line formulation of MPC. Most of the optimization problems are solved off-line so the proposed MPC algorithm is applicable to fast systems. This paper is organized as follows. The problem statement is presented in Section 2. Derivation of LMI constraints is presented in Section 3. In Section 4, an off-line robust MPC algorithm is proposed. In Section 5, the effectiveness of the proposed MPC algorithm is illustrated by two examples. Finally, the conclusions are drawn in Section 6.

Notation. For any vector $x$ and positive-definite matrix $P$, $\|x\|_{P}^{2}=x^{T} P x . x(k)$ is the state measured at real time $k$ and $x(k+i \mid k)$ is the state at prediction time $k+i$ predicted at real time $k$. The symbol $*$ denotes symmetric blocks in matrices. An element belonging to a convex hull $\mathrm{Co}\{\cdot\}$ means that it is a convex combination of the elements in $\{\cdot\}$. The time dependence $(k)$ of the MPC decision variables is often dropped for simplicity. $I$ is the identity matrix with appropriate dimension.

\section{Problem Statement}

Consider the following linear time-varying system:

$$
\begin{gathered}
x(k+1)=A(k) x(k)+B(k) u(k)+D(k) v(k) \\
y(k)=C x(k),
\end{gathered}
$$

where $x(k) \in \mathfrak{R}^{n_{x}}$ is the state, $u(k) \in \mathfrak{R}^{n_{u}}$ is the control input, $v(k) \in \mathfrak{R}^{n_{v}}$ is the disturbance, and $y(k) \in \mathfrak{R}^{n_{y}}$ is the output. The superscripts $n_{x}, n_{u}, n_{v}$, and $n_{y}$ are the number of elements in $x(k), u(k), v(k)$, and $y(k)$, respectively. The input and output constraints are

$$
\begin{aligned}
& \left|u_{h}(k)\right| \leq \bar{u}_{h}, \quad \bar{u}_{h}>0, h \in\left\{1,2, \ldots, n_{u}\right\}, \\
& \left|y_{r}(k)\right| \leq \bar{y}_{r}, \quad \bar{y}_{r}>0, r \in\left\{1,2, \ldots, n_{y}\right\},
\end{aligned}
$$

where $\bar{u}_{h}$ are the bounds of the elements $u_{h}(k)$ in the control input $u(k)$ and $\bar{y}_{r}$ are the bounds of the elements $y_{r}(k)$ in the output $y(k)$. It is assumed that any $A(k), B(k)$, and $D(k)$ belong to a convex polytope $\Omega_{A B D}$ defined by

$$
\begin{gathered}
\Omega_{A B D}=\operatorname{Co}\left\{\left[A_{1}, B_{1}, D_{1}\right],\left[A_{2}, B_{2}, D_{2}\right], \ldots,\right. \\
\left.\left[A_{n_{A B D}}, B_{n_{A B D}}, D_{n_{A B D}}\right]\right\} \\
{[A(k), B(k), D(k)]=\sum_{j=1}^{n_{A B D}} \alpha_{j}(k)\left[A_{j}, B_{j}, D_{j}\right],}
\end{gathered}
$$

where $\left[A_{j}, B_{j}, D_{j}\right]$ are the vertices of $\Omega_{A B D}, n_{A B D}$ is the number of the vertices of $\Omega_{A B D}$, and $\alpha_{j}(k)$ are the uncertain time-varying parameters whose sum is equal to 1 . The disturbance $v(k)$ is persistent and bounded. It is assumed that $v(k)$ belongs to an ellipsoid $\varepsilon_{v}$ associated with a symmetric positive-definite matrix $P_{v}$. Consider

$$
v(k) \in \varepsilon_{v}=\left\{v \mid\|v\|_{P_{v}}^{2} \leq 1\right\} .
$$

The objective is to find a state feedback control law $u(k+i \mid$ $k)=F x(k+i \mid k)$ that is able to guarantee both robust stability and constraint satisfaction within a positively invariant set.

Definition 1. The set $Z$ is said to be positively invariant set if it has the property that whenever the current state is contained in this set $x(k) \in Z$, all possible predicted states must be contained in this set $x(k+i \mid k) \in Z$ for all admissible realizations of $\alpha_{j}(k+i)$ and $v(k+i), i \geq 0$.

Remark 2. From the notion of quadratic boundedness [22], the system (1) is quadratically bounded with a common Lyapunov matrix $Q^{-1}$ and the ellipsoid $\varepsilon_{Q^{-1}}=\left\{x \in \mathfrak{R}^{n_{x}} /\|x\|_{Q^{-1}}^{2} \leq\right.$ 
$1\}$ is a positively invariant set if $\|x(k+i \mid k)\|_{Q^{-1}}^{2} \geq 1$ implies $\|x(k+i+1 \mid k)\|_{\mathrm{Q}^{-1}}^{2} \leq\|x(k+i \mid k)\|_{\mathrm{Q}^{-1}}^{2}$.

Consider the linear time-varying systems (1) to (5) at each sampling time $k$; a state feedback control law $u(k+i \mid$ $k)=F x(k+i \mid k)$ that guarantees both robust stability and constraint satisfaction within a positively invariant set $\varepsilon_{Q^{-1}}$ is obtained by solving the following optimization problem:

$$
\min _{\gamma, F, Q} \gamma
$$

s.t.

$$
\begin{gathered}
x(k+i+1 \mid k)=[A(k+i)+B(k+i) F] x(k+i \mid k) \\
+D(k+i) v(k+i), \quad x(k \mid k)=x(k), \\
\|x(k)\|_{Q^{-1}}^{2} \leq 1,
\end{gathered}
$$

$$
\begin{aligned}
& \text { If }\|x(k+i \mid k)\|_{Q^{-1}}^{2} \geq 1, \\
& \text { then }\|x(k+i+1 \mid k)\|_{Q^{-1}}^{2}-\|x(k+i \mid k)\|_{Q^{-1}}^{2} \\
& \quad \leq-\frac{1}{\gamma}\left[\|x(k+i \mid k)\|_{\psi}^{2}+\|F x(k+i \mid k)\|_{\sigma}^{2}\right], \\
& \left|u_{h}(k+i \mid k)\right| \leq \bar{u}_{h}, \quad \bar{u}_{h}>0, h \in\left\{1,2, \ldots, n_{u}\right\}, \\
& \left|y_{r}(k+i+1 \mid k)\right| \leq \bar{y}_{r}, \quad \bar{y}_{r}>0, r \in\left\{1,2, \ldots, n_{y}\right\},
\end{aligned}
$$

where $\gamma$ is the upper bound on $\sum_{i=0}^{\infty}\left[\|x(k+i \mid k)\|_{\psi}^{2}+\right.$ $\|K x(k+i \mid k)\|_{\sigma}^{2}$ ] (the proof details can be found in [6]) and $\psi$ and $\sigma$ are symmetric weighting matrices. A positively invariant set containing the measured state at each sampling time is computed by (8). All possible predicted states are restricted to lying in a positively invariant set by (9). The input and output constraints are guaranteed by (10) and (11), respectively.

\section{Derivation of LMI Constraints}

Proposition 3 (robust stability). Equation (9) is satisfied if there exist matrices $Y, Q$, a scalar $\gamma$, and a scalar $0<\theta<1$ such that the following LMIs are satisfied:

$$
\begin{gathered}
{\left[\begin{array}{ccccc}
(1-\theta) Q & * & * & * & * \\
\left(A_{j} Q+B_{j} Y\right) & Q & * & * & * \\
\sigma^{1 / 2} Y & 0 & \gamma I & * & * \\
\psi^{1 / 2} Q & 0 & 0 & \gamma I & * \\
\left(A_{j} Q+B_{j} Y\right) & 0 & 0 & 0 & S-Q
\end{array}\right], \quad j \in\left\{1,2, \ldots, n_{A B D}\right\}} \\
\\
{\left[\begin{array}{ccc}
S & * \\
Q & D_{j}\left(\theta P_{v}\right)^{-1} D_{l}^{T}
\end{array}\right] \geq 0} \\
j \in\left\{1,2, \ldots, n_{A B D}\right\}, \quad l \in\left\{1,2, \ldots, n_{A B D}\right\}
\end{gathered}
$$

where the state feedback gain is parameterized as $F=$ $Y Q^{-1}$. Then, all possible predicted states are restricted to lying in a positively invariant set by (9). A positively invariant set containing the measured state at each sampling time is computed by (8) which is equivalent to the following LMI:

$$
\left[\begin{array}{cc}
1 & * \\
x(k) & Q
\end{array}\right] \geq 0
$$

Proof. From $\|x(k+i \mid k)\|_{Q^{-1}}^{2} \geq 1$ and $\|v(k+i)\|_{P_{y}}^{2} \leq 1$, (9) is equivalent to

$$
\begin{aligned}
\|v(k+i)\|_{P_{v}}^{2} \leq & \|x(k+i \mid k)\|_{Q^{-1}}^{2} \Longrightarrow\|x(k+i+1 \mid k)\|_{Q^{-1}}^{2} \\
& -\|x(k+i \mid k)\|_{Q^{-1}}^{2} \\
\leq & -\frac{1}{\gamma}\left[\|x(k+i \mid k)\|_{\psi}^{2}+\|F x(k+i \mid k)\|_{\sigma}^{2}\right] .
\end{aligned}
$$

By substituting (7), (14) can be written as

$$
\begin{aligned}
& {\left[\begin{array}{c}
x(k+i \mid k) \\
v(k+i)
\end{array}\right]^{T}\left[\begin{array}{cc}
Q^{-1} & 0 \\
0 & -P_{v}
\end{array}\right]\left[\begin{array}{c}
x(k+i \mid k) \\
v(k+i)
\end{array}\right] \geq 0} \\
& \Longrightarrow\left[\begin{array}{c}
x(k+i \mid k) \\
v(k+i)
\end{array}\right]^{T} \\
& \times\left[\begin{array}{c}
Q^{-1}-(A(k+i)+B(k+i) F)^{T} Q^{-1}(A(k+i)+B(k+i) F) \\
-\frac{F^{T} \sigma F}{\gamma}-\frac{\psi}{\gamma}
\end{array}\right]\left[\begin{array}{c}
x(k+i \mid k) \\
v(k+i)
\end{array}\right] \geq 0 .
\end{aligned}
$$




$$
\left[\begin{array}{cc}
Q^{-1}-(A(k+i)+B(k+i) F)^{T} Q^{-1}(A(k+i)+B(k+i) F) & \\
-\frac{F^{T} \sigma F}{\gamma}-\frac{\psi}{\gamma} & * \\
-D(k+i)^{T} Q^{-1}(A(k+i)+B(k+i) F) & -D(k+i)^{T} Q^{-1} D(k+i)
\end{array}\right] \geq \theta\left[\begin{array}{cc}
Q^{-1} & 0 \\
0 & -P_{v}
\end{array}\right]
$$

By applying the Schur complement, (16) is equivalent to

$$
\begin{aligned}
Q^{-1}- & (A(k+i)+B(k+i) F)^{T} Q^{-1}(A(k+i)+B(k+i) F) \\
- & \frac{F^{T} \sigma F}{\gamma}-\frac{\psi}{\gamma}-\theta Q^{-1}-(A(k+i)+B(k+i) F)^{T} \\
& \times\left[Q D(k+i)^{-T} \theta P_{v} D(k+i)^{-1} Q-Q\right]^{-1} \\
& \times(A(k+i)+B(k+i) F) \geq 0 .
\end{aligned}
$$

Premultiplying by $Q^{T}$, postmultiplying by $Q$, and applying the Schur complement lead to (12).

Proposition 4 (input constraint satisfaction). The input constraint (10) is satisfied if there exist matrices $Y$ and $Q$ such that the following LMIs are satisfied:

$$
\left[\begin{array}{cc}
\chi & * \\
Y^{T} & \mathrm{Q}
\end{array}\right] \geq 0, \quad \chi_{h h} \leq \bar{u}_{h}^{2}, h \in\left\{1,2, \ldots, n_{u}\right\} .
$$

Proof. By defining $\zeta_{h}$ as the $h$ th row of the $n_{u}$-dimensional identity matrix, we can see that

$$
\begin{aligned}
\max _{i \geq 0}\left|\zeta_{h} u(k+i \mid k)\right|^{2} & =\max _{i \geq 0}\left|\zeta_{h} F x(k+i \mid k)\right|^{2} \\
& \leq \max _{i \geq 0}\left\|\zeta_{h} F x(k+i \mid k)\right\|^{2} .
\end{aligned}
$$

Substituting $F=Y Q^{-1}$ leads to

$$
\max _{i \geq 0}\left|\zeta_{h} u(k+i \mid k)\right|^{2} \leq \max _{i \geq 0}\left\|\zeta_{h} Y Q^{-1} x(k+i \mid k)\right\|^{2} .
$$

Since $\|x(k+i \mid k)\|_{\mathrm{Q}^{-1}}^{2} \leq 1$, it is seen that

$$
\max _{i \geq 0}\left|\zeta_{h} u(k+i \mid k)\right|^{2} \leq\left\|\zeta_{h} Y Q^{-1 / 2}\right\|^{2} .
$$

The input constraint (10) is satisfied if

$$
\left\|\zeta_{h} Y Q^{-1 / 2}\right\|^{2} \leq \bar{u}_{h}^{2}
$$

By applying the Schur complement, (22) is equivalent to (18).
Proposition 5 (output constraint satisfaction). The output constraint (11) is satisfied if there exist matrices $Y$ and $Q$ such that the following LMIs are satisfied:

$$
\begin{gathered}
{\left[\begin{array}{ccc}
\frac{\Gamma}{2} & * & * \\
\left(A_{j} Q+B_{j} Y\right)^{T} C^{T} & Q & * \\
D_{j}^{T} C^{T} & 0 & P_{v}
\end{array}\right] \geq 0,} \\
\Gamma_{r r} \leq \bar{y}_{r}^{2}, \quad r \in\left\{1,2, \ldots, n_{y}\right\}, \quad j \in\left\{1,2, \ldots, n_{A B D}\right\} .
\end{gathered}
$$

Proof. By defining $\zeta_{r}$ as the $r$ th row of the $n_{y}$-dimensional identity matrix, we can see that

$$
\begin{aligned}
\max _{i \geq 0}\left|\zeta_{r} y(k+i+1 \mid k)\right|^{2} & =\max _{i \geq 0}\left|\zeta_{r} C x(k+i+1 \mid k)\right|^{2} \\
& \leq \max _{i \geq 0}\left\|\zeta_{r} C x(k+i+1 \mid k)\right\|^{2} .
\end{aligned}
$$

Substituting (7) and applying $F=Y Q^{-1}$ lead to

$$
\begin{aligned}
\max _{i \geq 0}\left|\zeta_{r} y(k+i+1 \mid k)\right|^{2} \\
\leq \max _{i \geq 0} \| \zeta_{r} C\left[\left(A(k+i)+B(k+i) Y Q^{-1}\right) D(k+i)\right] \\
\times\left[\begin{array}{c}
x(k+i \mid k) \\
v(k+i)
\end{array}\right] \|^{2} .
\end{aligned}
$$

Since $\|x(k+i \mid k)\|_{Q^{-1}}^{2} \leq 1$ and $\|v(k+i \mid k)\|_{P_{v}}^{2} \leq 1$, it is seen that

$$
\begin{aligned}
\max _{i \geq 0}\left|\zeta_{r} y(k+i+1 \mid k)\right|^{2} \\
\leq 2 \| \zeta_{r} C[(A(k+i) Q+B(k+i) Y) D(k+i)] \\
\times\left[\begin{array}{cc}
Q^{-1 / 2} & 0 \\
0 & P_{v}^{-1 / 2}
\end{array}\right]\|\|^{2}
\end{aligned}
$$


The output constraint (11) is satisfied if

$$
\begin{gathered}
2 \| \zeta_{r} C[(A(k+i) Q+B(k+i) Y) D(k+i)] \\
\times\left[\begin{array}{cc}
Q^{-1 / 2} & 0 \\
0 & P_{v}^{-1 / 2}
\end{array}\right] \|^{2} \leq \bar{y}_{r}^{2} .
\end{gathered}
$$

By applying the Schur complement, (27) is equivalent to (23).

\section{An Off-Line Formulation of Robust MPC}

By considering Propositions 3, 4, and 5, a state feedback control law that guarantees both robust stability and constraint satisfaction can be calculated.

Theorem 6. Consider the linear time-varying systems (1) to (5) at each sampling time $k$; a state feedback control law $u(k+$ $i \mid k)=F x(k+i \mid k), F=Y Q^{-1}$, that guarantees both robust stability and constraint satisfaction within a positively invariant set $\varepsilon_{Q^{-1}}$ is obtained by solving the following optimization problem:

$$
\begin{aligned}
& \min _{\gamma, Y, Q, \theta} \gamma \\
& \text { s.t. } \quad(12),(13),(18),(23)
\end{aligned}
$$

Proof. The proof follows Propositions 3, 4, and 5.

The optimization problem (28) can be solved by LMI techniques and line search of $\theta$ over the interval $(0,1)$. However, it is computationally demanding to solve the optimization problem (28) at each sampling time. Inspired by Wan and Kothare [20], we propose an off-line robust MPC algorithm that solves the optimization problem off-line.

\section{Algorithm 7.}

Off-Line. Choose a sequence of states $x_{i}, i \in\{1,2, \ldots, N\}$. For each $i$, substitute $x(k)$ in (13) by $x_{i}$ and solve the optimization problem (28) to obtain a sequence of state feedback gains $F_{i}$ and a sequence of positively invariant sets $\varepsilon_{Q_{i}^{-1}}=\{x \in$ $\left.\Re^{n_{x}} / x^{T} Q_{i}^{-1} x \leq 1\right\}$. Then, store $F_{i}$ and $Q_{i}^{-1}$ in a look-up table.

Remark 8. A sequence of states $x_{i}, i \in\{1,2, \ldots, N\}$ should be chosen such that the positively invariant sets $\varepsilon_{\mathrm{Q}_{i}^{-1}}$ obtained from solving the optimization problem (28) are nested $\left(\varepsilon_{Q_{i+1}^{-1}} \subset \varepsilon_{Q_{i}^{-1}}, Q_{i+1}^{-1}>Q_{i}^{-1}, \forall i \neq N\right)$. This is to guarantee that the state of the process is kept within $\varepsilon_{Q_{i}^{-1}}$ and driven towards $\varepsilon_{Q_{i+1}^{-1}}$, and so on. For the ease of implementation, we can choose an arbitrary one-dimensional subspace and discretize it to construct a set of discrete points.

Remark 9. The number of the chosen states $N$ (or, equivalently, the number of the positively invariant sets) affects the control performance. Although the state feedback gain $F_{i}$ can guarantee robust stability, it is not necessary to keep this state feedback gain constant. By increasing $N$, the control performance is improved due to the fact that we have more freedom to adopt varying feedback gains based on the distance between the state and the origin. A larger $N$ implies better control performance at the price of a higher off-line computational load so a suitable tradeoff is required in practice.

On-Line. At each sampling time $k$, measure the state $x(k)$. Then, perform a bisection search over $Q_{i}^{-1}$ in the lookup table to find the largest $i$ such that $x(k)^{T} Q_{i}^{-1} x(k) \leq$ 1 or, equivalently, the smallest positively invariant set $\varepsilon_{Q_{i}^{-1}}$ containing the measured state $x(k)$. Apply the corresponding state feedback control law $u(k)=F_{i} x(k)$ to the process.

The satisfaction of (9) for each $F_{i}$ ensures robust stability within a positively invariant set $\varepsilon_{Q_{i}^{-1}}$. Given an initial state satisfying $x(0)^{T} Q_{1}^{-1} x(0) \leq 1$, robust stability is ensured by applying Algorithm 7.

\section{Examples}

The numerical simulations have been performed in Intel Core 2 Duo (2.53 GHz), 2 GB RAM, using SeDuMi [23] and YALMIP [24] within the Matlab R2008a environment.

Example 1. The first example is an angular positioning system adapted from Kothare et al. [6]. The system consists of an electric motor driving a rotating antenna so that it always points in the direction of a moving object. The motion of the antenna can be described by the following linear timevarying system:

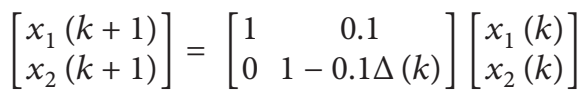

$$
\begin{aligned}
& +\left[\begin{array}{c}
0 \\
0.0787
\end{array}\right] u(k)+\left[\begin{array}{l}
0.15 \\
0.15
\end{array}\right] v(k) \text {, } \\
& y(k)=\left[\begin{array}{ll}
1 & 0 \\
0 & 0
\end{array}\right]\left[\begin{array}{l}
x_{1}(k) \\
x_{2}(k)
\end{array}\right],
\end{aligned}
$$

where $x_{1}(k)$ is the angular position of the antenna, $x_{2}(k)$ is the angular velocity of the antenna, $u(k)$ is the input voltage to the motor, $\Delta(k)$ is the uncertain time-varying parameter which is proportional to the coefficient of viscous friction in the rotating parts, and $v(k)$ is the disturbance acting on the system. Since $0.1 \leq \Delta(k) \leq 10$, we can see that

$$
A(k) \in \operatorname{Co}\left\{A_{1}, A_{2}\right\} \text {, }
$$

where

$$
A_{1}=\left[\begin{array}{cc}
1 & 0.1 \\
0 & 0.99
\end{array}\right], \quad A_{2}=\left[\begin{array}{cc}
1 & 0.1 \\
0 & 0
\end{array}\right] .
$$

The objective is to robustly stabilize $x_{1}(k)$ by manipulating $u(k)$. The input constraint is $|u(k)| \leq 2$ volts. The symmetric weighting matrices in (9) are

$$
\Psi=\left[\begin{array}{ll}
1 & 0 \\
0 & 0
\end{array}\right], \quad \sigma=0.00002 .
$$

A sampling period is $0.1 \mathrm{~s}$. 


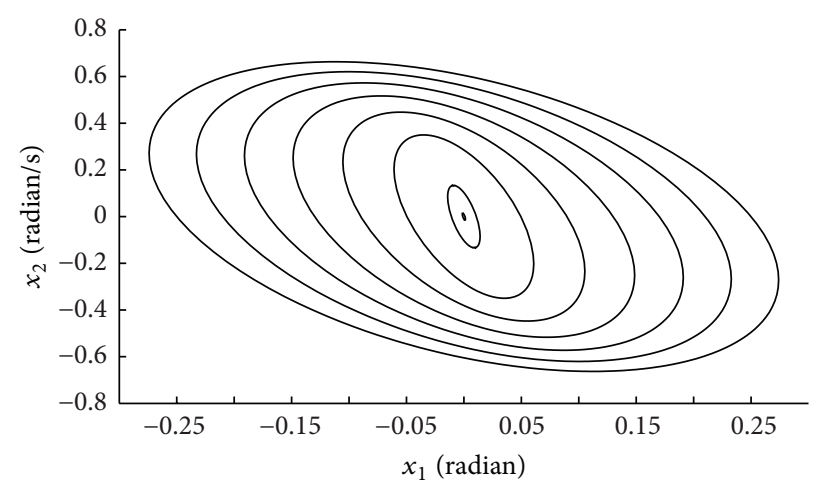

FIGURE 1: The positively invariant sets computed off-line in Example 1.
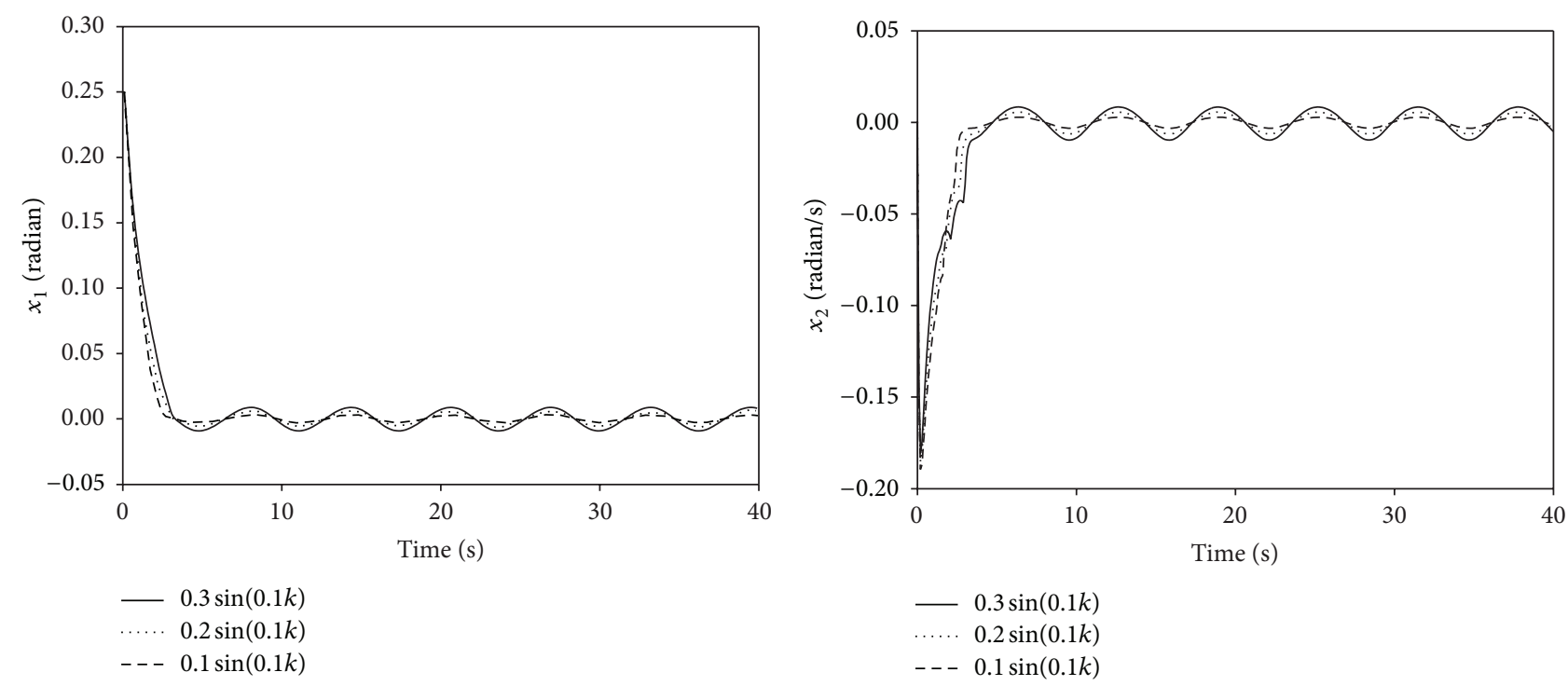

(a) Regulated output

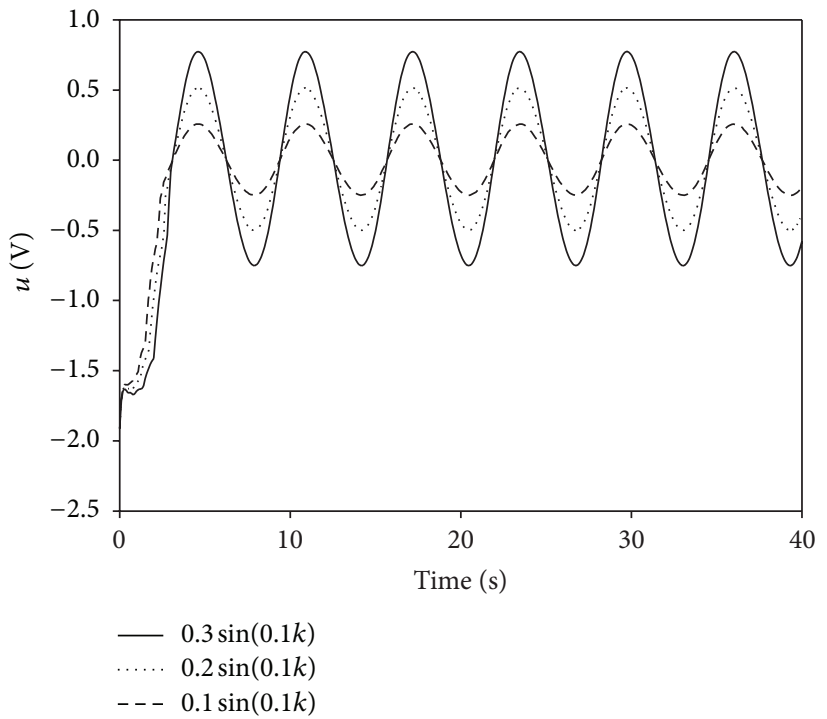

(b) Control input

FIGURE 2: The closed-loop responses of the system in Example 1 (a) regulated output and (b) control input. 
Figure 1 shows the positively invariant sets $\varepsilon_{\mathrm{Q}_{i}^{-1}}, i=$ $\{1,2, \ldots, 8\}$, computed off-line by Algorithm 7. In this example, the values of state feedback gains $F_{i}$ are almost constant beyond $i=8$ so only eight positively invariant sets are computed off-line.

Figure 2 shows the closed-loop responses of the system when the uncertain time-varying parameter is varied as $\Delta(k)=4.95 \sin (0.1 k)+5.05$ and the disturbance is varied as $v(k)=0.3 \sin (0.1 k), 0.2 \sin (0.1 k)$, and $0.1 \sin (0.1 k)$, respectively. It can be observed that $x_{1}$ and $x_{2}$ are bounded for all values of uncertain time-varying parameter and disturbance so robust stability is ensured by applying Algorithm 7. The off-line computational time is $2.938 \mathrm{sec}$. The on-line computational time is very small $(<0.001 \mathrm{sec}$ per sampling period $)$ since all of the optimization problems are solved off-line.

Example 2. Consider the following linear time-varying system adapted from Mayne et al. [25]:

$$
\begin{gathered}
{\left[\begin{array}{l}
x_{1}(k+1) \\
x_{2}(k+1)
\end{array}\right]=\left[\begin{array}{ll}
1 & 1 \\
0 & 1
\end{array}\right]\left[\begin{array}{l}
x_{1}(k) \\
x_{2}(k)
\end{array}\right]+\left[\begin{array}{l}
1 \\
1
\end{array}\right] u(k)+\left[\begin{array}{l}
1 \\
1
\end{array}\right] v(k)} \\
y(k)=\left[\begin{array}{ll}
1 & 0 \\
0 & 1
\end{array}\right]\left[\begin{array}{l}
x_{1}(k) \\
x_{2}(k)
\end{array}\right]
\end{gathered}
$$

where $v(k)=0.1 \sin (0.1 k)$ is the disturbance acting on the system. The objective is to robustly stabilize $x_{1}(k)$ and $x_{2}(k)$ by manipulating $u(k)$. The input constraint is $|u(k)| \leq 3$. The symmetric weighting matrices in (9) are

$$
\Psi=\left[\begin{array}{ll}
1 & 0 \\
0 & 1
\end{array}\right], \quad \sigma=0.01 .
$$

Figure 3 shows the positively invariant sets $\varepsilon_{Q_{i}^{-1}}, i=$ $\{1,2, \ldots, 9\}$, computed off-line by Algorithm 7. In this example, the values of state feedback gains $F_{i}$ are almost constant beyond $i=9$, so only nine positively invariant sets are computed off-line.

Figure 4 shows the state trajectories evolving from the initial point $(1.6,-1.6)$. It can be observed that Algorithm 7 gives nearly the same control performance as Theorem 6 (online algorithm) while no optimization problems need to be solved on-line. This is due to the fact that, for the proposed off-line algorithm, the state feedback gain is updated at each sampling time based on the distance between the measured state and the origin.

\section{Conclusions}

In this research, we have presented an off-line robust constrained MPC algorithm for linear time-varying systems. Both model uncertainty and bounded additive disturbance are explicitly taken into account in the off-line formulation of MPC. All of the optimization problems are solved off-line so the proposed MPC algorithm is applicable to fast systems. Robust stability and constraint satisfaction are guaranteed by applying the proposed algorithm.

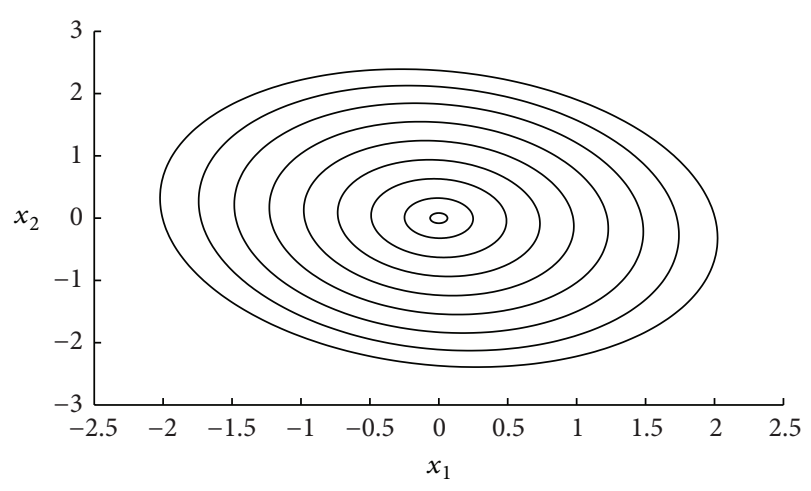

FIGURE 3: The positively invariant sets computed off-line in Example 2.

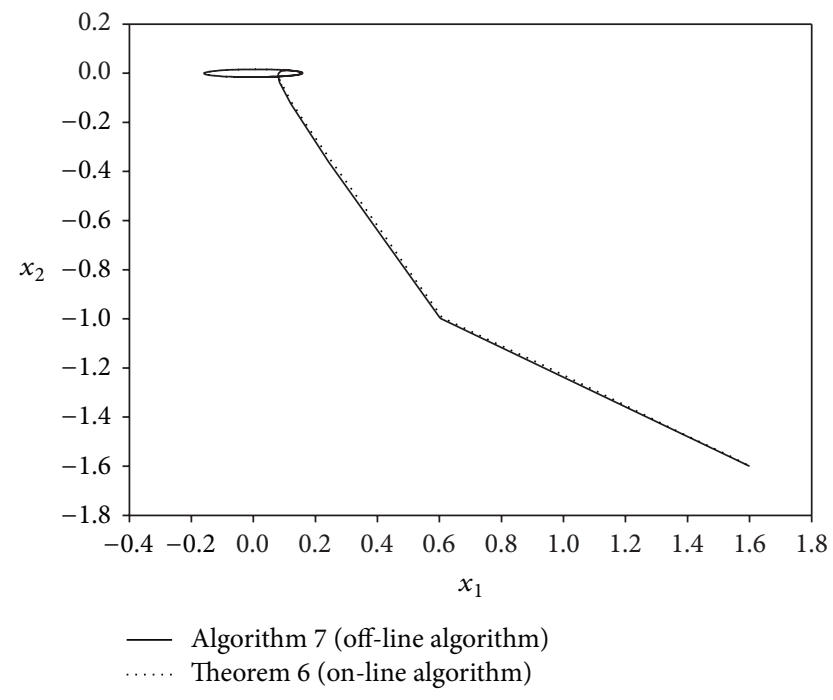

FIgURE 4: The state trajectories in Example 2.

\section{Conflict of Interests}

The authors declare that there is no conflict of interests regarding the publication of this paper.

\section{Acknowledgment}

This research project is supported by Mahidol University.

\section{References}

[1] J. H. Lee, "Model predictive control: review of the three decades of development," International Journal of Control, Automation and Systems, vol. 9, no. 3, pp. 415-424, 2011.

[2] W. Al-Gherwi, H. Budman, and A. Elkamel, "Robust distributed model predictive control: a review and recent developments," Canadian Journal of Chemical Engineering, vol. 89, no. 5, pp. 1176-1190, 2011.

[3] J. B. Rawlings and D. Q. Mayne, Model Predictive Control: Theory and Design, Nob Hill, Madison, Wis, USA, 1st edition, 2009.

[4] S. J. Qin and T. A. Badgwell, "A survey of industrial model predictive control technology," Control Engineering Practice, vol. 11, no. 7, pp. 733-764, 2003. 
[5] D. Q. Mayne, J. B. Rawlings, C. V. Rao, and P. O. M. Scokaert, "Constrained model predictive control: stability and optimality," Automatica, vol. 36, no. 6, pp. 789-814, 2000.

[6] M. V. Kothare, V. Balakrishnan, and M. Morari, "Robust constrained model predictive control using linear matrix inequalities," Automatica, vol. 32, no. 10, pp. 1361-1379, 1996.

[7] S. Boyd and L. Vandenberghe, Convex Optimization, Cambridge University Press, Cambridge, Cambridge, Mass, USA, 1st edition, 2004.

[8] W.-J. Mao, "Robust stabilization of uncertain time-varying discrete systems and comments on an improved approach for constrained robust model predictive control," Automatica, vol. 39, no. 6, pp. 1109-1112, 2003.

[9] N. Wada, K. Saito, and M. Saeki, "Model predictive control for linear parameter varying systems using parameter dependent Lyapunov function," IEEE Transactions on Circuits and Systems, vol. 53, no. 12, pp. 1446-1450, 2006.

[10] A. C. Brooms, B. Kouvaritakis, and Y. I. Lee, "Constrained MPC for uncertain linear systems with ellipsoidal target sets," Systems \& Control Letters, vol. 44, no. 3, pp. 157-166, 2001.

[11] Y. I. Lee and B. Kouvaritakis, "Robust receding horizon predictive control for systems with uncertain dynamics and input saturation," Automatica, vol. 36, no. 10, pp. 1497-1504, 2000.

[12] J. A. Rossiter, B. Kouvaritakis, and M. Bacic, "Interpolation based computationally efficient predictive control," International Journal of Control, vol. 77, no. 3, pp. 290-301, 2004.

[13] B. Pluymers, J. A. Rossiter, J. A. K. Suykens, and B. De Moor, "The efficient computation of polyhedral invariant sets for linear systems with polytopic uncertainty," in Proceedings of the American Control Conference (ACC '05), pp. 804-809, Portland, Ore, USA, June 2005.

[14] P. Bumroongsri and S. Kheawhom, "An off-line robust MPC algorithm for uncertain polytopic discrete-time systems using polyhedral invariant sets," Journal of Process Control, vol. 22, no. 6, pp. 975-983, 2012.

[15] A. Luca, P. Rodriguez-Ayerbe, D. Dumur, and P. Lefranc, "Invariant sets techniques for Buck DC-DC converter control," in Proceedings of the 11th International Conference on Control, Automation, Robotics and Vision (ICARCV '10), pp. 1917-1922, December 2010.

[16] A. Luca, P. Rodriguez-Ayerbe, D. Dumur, and P. Lefranc, "Buck DC-DC converter control using invariant sets techniques," in Proceedings of the 15th IEEE Mediterranean Electrotechnical Conference (MELECON '10), pp. 184-189, April 2010.

[17] W. Langson, I. Chryssochoos, S. V. Raković, and D. Q. Mayne, "Robust model predictive control using tubes," Automatica, vol. 40, no. 1, pp. 125-133, 2004.

[18] D. Limon, I. Alvarado, T. Alamo, and E. F. Camacho, "Robust tube-based MPC for tracking of constrained linear systems with additive disturbances," Journal of Process Control, vol. 20, no. 3, pp. 248-260, 2010.

[19] R. Gonzalez, M. Fiacchini, T. Alamo, J. L. Guzman, and F. Rodriguez, "Online robust tube-based MPC for time-varying systems: a practical approach," International Journal of Control, vol. 84, no. 6, pp. 1157-1170, 2011.

[20] Z. Wan and M. V. Kothare, "An efficient off-line formulation of robust model predictive control using linear matrix inequalities," Automatica, vol. 39, no. 5, pp. 837-846, 2003.

[21] B. Ding, Y. Xi, M. T. Cychowski, and T. O'Mahony, "Improving off-line approach to robust MPC based-on nominal performance cost," Automatica, vol. 43, no. 1, pp. 158-163, 2007.
[22] A. Alessandri, M. Baglietto, and G. Battistelli, "On estimation error bounds for receding-horizon filters using quadratic boundedness," IEEE Transactions on Automatic Control, vol. 49, no. 8, pp. 1350-1355, 2004.

[23] J. F. Sturm, "Using SeDuMi 1.02, a MATLAB toolbox for optimization over symmetric cones," Optimization Methods and Software, vol. 11-12, no. 1-4, pp. 625-653, 1999, Interior point methods.

[24] J. Löfberg, "Automatic robust convex programming," Optimization Methods \& Software, vol. 27, no. 1, pp. 115-129, 2012.

[25] D. Q. Mayne, M. M. Seron, and S. V. Raković, "Robust model predictive control of constrained linear systems with bounded disturbances," Automatica, vol. 41, no. 2, pp. 219-224, 2005. 


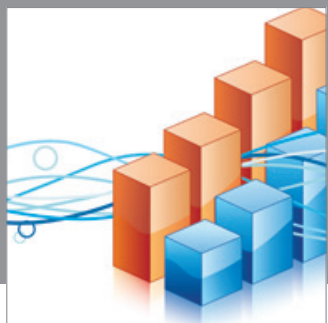

Advances in

Operations Research

mansans

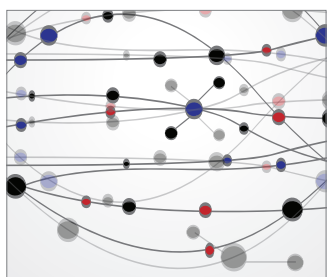

The Scientific World Journal
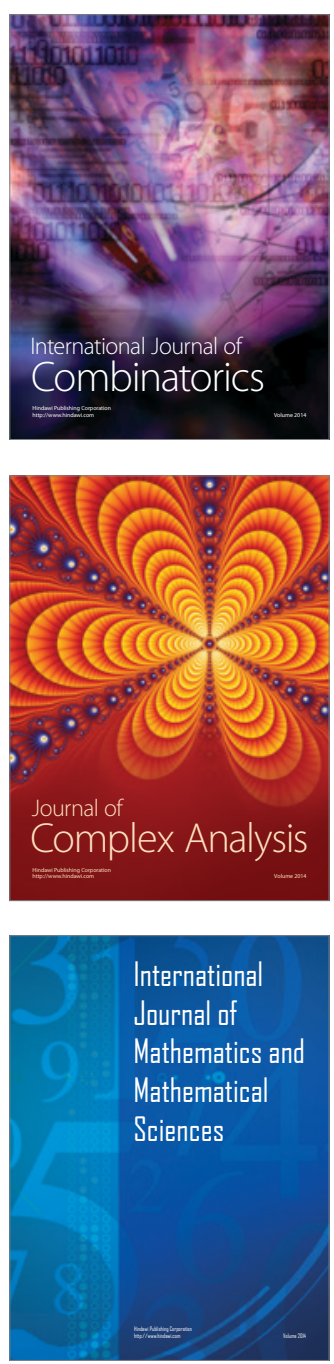
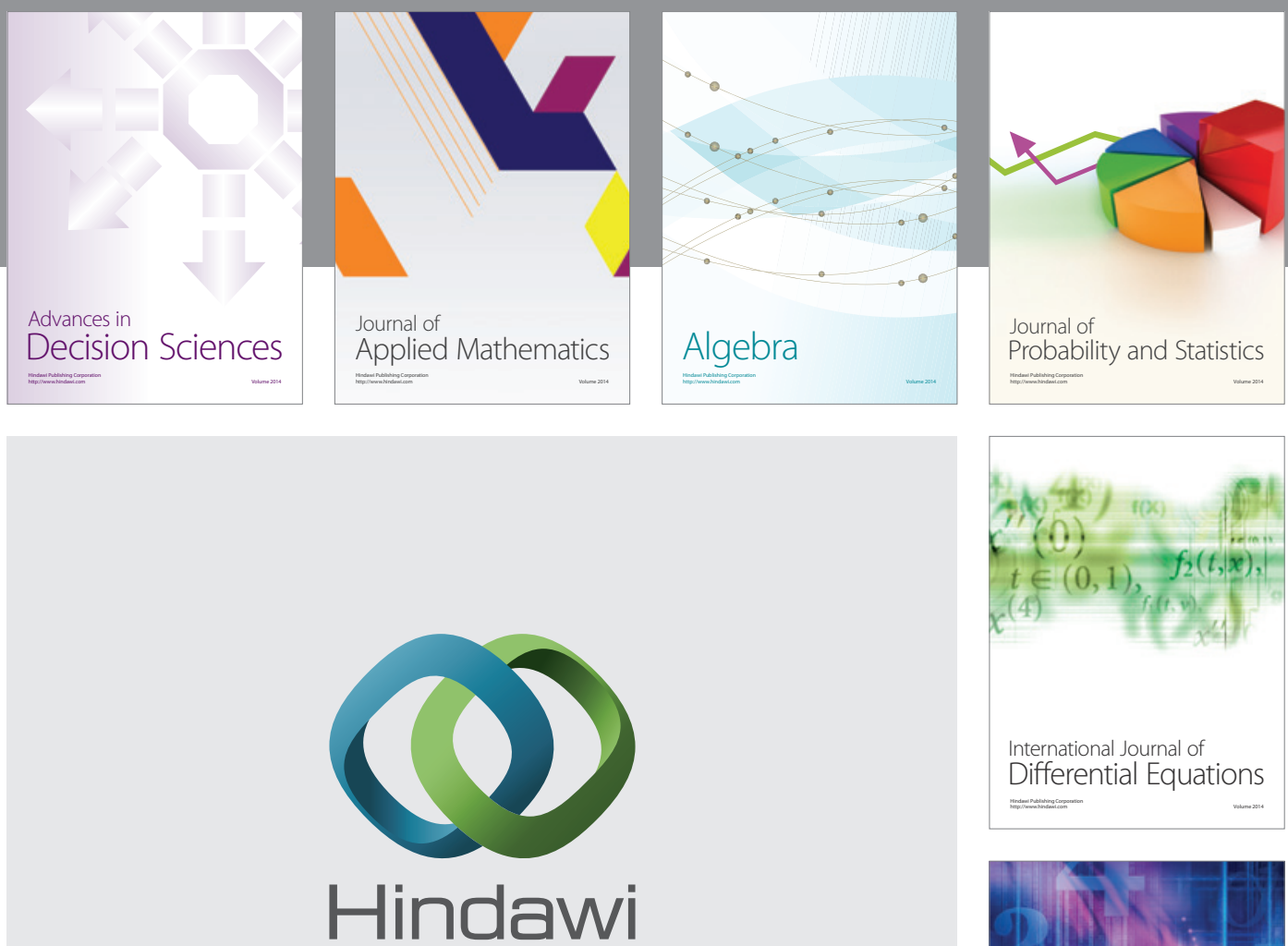

Submit your manuscripts at http://www.hindawi.com
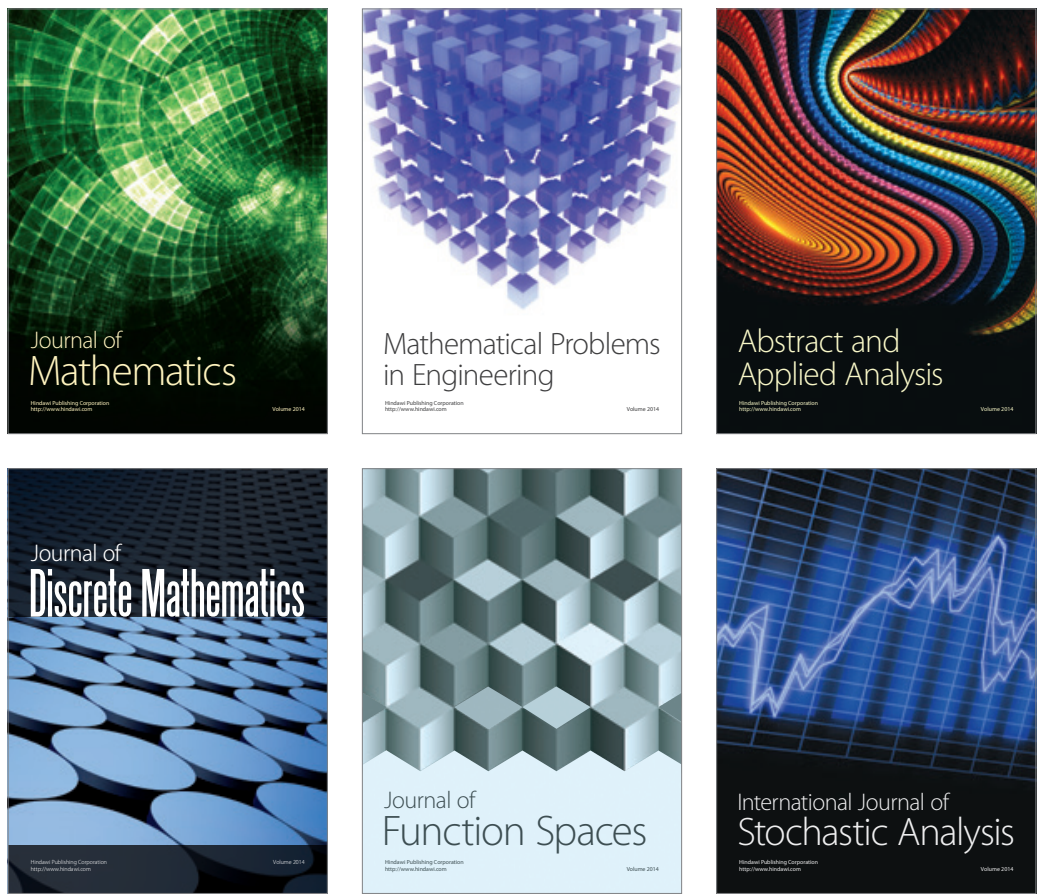

Journal of

Function Spaces

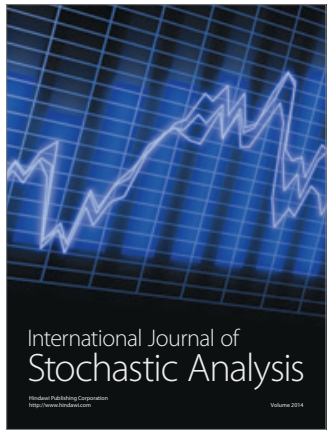

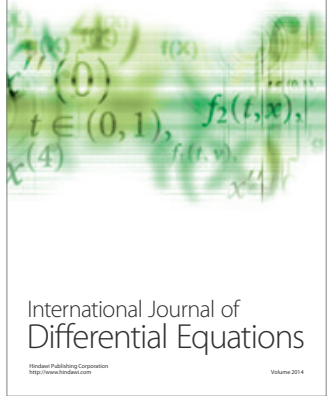
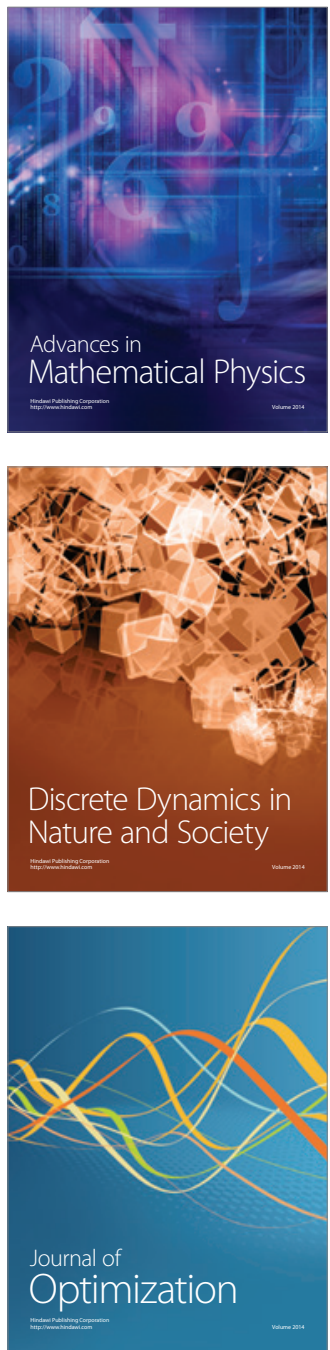